\title{
An Integrated Methodology of Manufacturing Business Improvement Strategies
}

\author{
S Berkhauer-Smith ${ }^{1}$ and $R$ Bhatti ${ }^{1}$ \\ 1 The School of Engineering, The University of Greenwich, Pembroke \\ Building, Central Avenue, Chatham Maritime, Chatham, Kent. ME4 4TB. \\ U.K. bsl2agreac.uk. $0044(0) 1634883473$.
}

\begin{abstract}
Business Environments need to react more efficiently and quickly to demand in today's global markets. Previous research by the authors entailed reviewing the current understanding of the different types of generic business improvements that are applicable to the production environment through a literature review. It highlighted that widely used methodologies have similarly links and differentiating characteristics, thus producing many types of implementation strategies. The research resulted in studying the interrelationships between these Manufacturing Business Improvement Concepts including Cultural Issues surrounding process improvement initiatives, so they can be unified into an integrated Methodology creating a unique strategy that can be correctly tailored to a chosen environment. This paper outlines a methodology that involves ten stages of change including the planning, creating, data collection, analysis and strategic implementation to apply. The approach flows through the change process systematically highlighting how to achieve the best outcome and feedback into the system is also visible.
\end{abstract}

\section{Keywords}

Process Improvement, Improving Operations, Integrated Strategy Application, Improvement Strategy Methodology, Manufacturing Operations.

\section{Introduction}

Research was initiated [2] by understanding currently practised Manufacturing Business Improvement Strategies centred around Lean Manufacture. This work studied the associations of these methods and the results highlighted that there are many techniques being applied and investigated at many different levels. From this 
there was a requirement to understand the cultural aspects surrounding these Manufacturing Business Improvements to eliminate the theory of " $85 \%$ of change projects fail" [6]. These writers also stated that;

....."That many such initiatives failed because they had concentrated on the purpose and ignores the people who made them work." [6]

This further research was undertaken and the results confirmed the need for an integrated methodology of both technical and cultural strategies that highlighted a "specific development path to follow" [3]. This research work was taken further and a comprehensive methodology was created to meet these requirements found from the previous work of the authors [4]. The hypothesis from the literature review created two main objectives to be tested within the methodology. This methodology satisfies the following two main objectives;

1) An Integrated Business Improvement system that analysed many improvement concepts.

2) Implementing this theoretical design through analysing and evolving the cultural aspects.

The main significant advantaged gained from the methodology is having a specific development path to follow.

\section{Methodology}

A methodology was created and consists of two parts; the Design Phase, which creates the project, collecting the information and analysing it. This information will be used to create the second part; the Implementation Phase. This element shall understand the strategy and the implementation of the theoretical design. The methodology will be used in case studies to secure a higher success rate of implementation and the continuation of improvement. The diagram, Fig. 1 highlights the stages of change; the planning, creating, data collection, analysis and strategic implementation to apply. The diagram also shows the process flow and how at some point the stages work in parallel to achieve the best outcome. The feedback into the system is also visible; it highlights how at every stage it is very important to feedback the information gained. This will ensure the project definition remains true to the project brief.

The stages created in Fig. 1 have been developed further to create a number of guidelines to enable greater use in cases to test the hypothesis. Each stage is designed so that there are a number of guidelines to follow and then within these guidelines there are suggestions on how to achieve these requirements. The methodology is colour coded to indicate different parts of the methodology; there is a distinction between those aspects that are planning, data collection and implementation, benchmarking therefore making statements and documenting and finalising those areas that require feedback loops. 


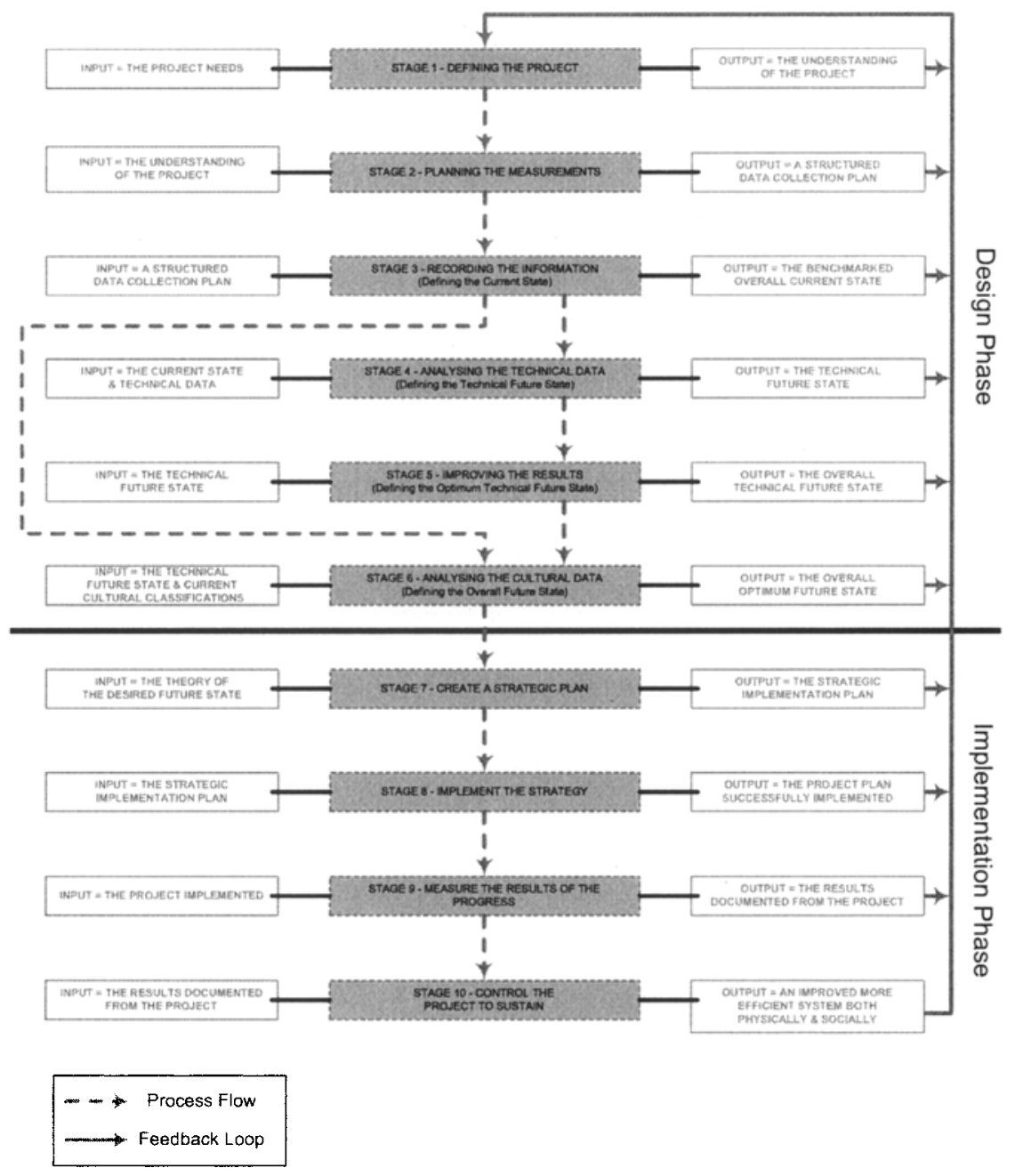

Fig. 1. The Process Flow of the Integrated Business Improvement Methodology.

\subsection{Design Phase}

This phase was created to guide the methodology through the different stages of the design of the project. It was also derived to highlight the importance of the integration of the current Business Improvement ideas and how different scenarios require different aspects of these concepts to be used. So it is not necessary to 
assume that one technique is applicable to all and so the art of this methodology is to have the ability to select what is suitable.

The First Stage of the methodology involves taking the project needs and defining them. Objectives as to why the project is needed are clarified and this will in turn define the strategy importance of the project within the business. It is also necessary at this stage to understand the project strategy; this would entail investigating the options and path ways of the project, to appreciate the future market share, as well as the desired outcome of the improvement process. Other areas that are required at this stage are the scope of the project, as well as the project due date and the deliverables. The outcome from this stage is the knowledge of the current flow of the business project. From this the measurements (Stage Two) that are required for the project can then be planned. A feedback loop into the system is essential to ensure that the project remains true to the brief. It also ensures that the stakeholders are happy with the monitored progress.

The Second Stage of the improvement process is to plan the measurements that are required. As the project is defined and the scope of the project analysed, a plan of the data to be measured should be calculated. It should also involve how these measurements will be collected, by whom and in what format, so that the results can easily be analysed.

This stage is the most significant part of the methodology and this highlights the originality of the research. An important factor of this stage is the integration of the Operational Business Improvements used in many businesses, mainly manufacturing. All of the key business improvement concepts are evaluated for their suitability to the project. This methodology incorporates a checklist facility that enables the user to select the specifics required, through a weighted method, therefore directing the pathway of the development. It is expected that in most cases not all techniques will be applicable; however it is thought that it will be necessary at some point in the transformation process.

By using this method of analysis the user will gain a better understanding of the project requirements as well as the improvement techniques. The integrated system will uniquely prioritise the amalgamated changes suggested, therefore creating a direction of physical change. From this stage a detailed data collection plan can be created. The research hypothesis is tested to understand how to fully integrate these concepts to absorb the better qualities of each technique, to create a fully improved system specific to the project. The Third Stage requires all of the information planned to be collected through the structured data collection plan that was derived within Stage Two of the methodology. The outcome from this Third Stage will be the overall current state of the project.

It is here in the improvement process that the methodology becomes divided; the cultural issues concerned with improvements do not exist without the technical changes forming. In this approach the technical advances will be documented theoretically and then analysed. Thus, allowing these technical aspects to be involved when deriving the cultural future state, this also allows greater change of successful implementation. So after technical issues have been assessed, these ideas influence the cultural changes required and so both features have then fused together to create a unified methodology, that will be introduced into the old system together. 
The Fourth Stage uses only the technical data for analyse, from this benchmarked current state the information is analysed by using the Improvement Strategies. This transforms the model into the Technical Future State. In turn these technical changes should be documented for investigation, by highlighting the significant technical changes. With this benchmarked technical future state vision, the methodology creates questions to be answered about the reliability and achievability of the results produced from the technical theory. The approach that the methodology uses to accomplish this is through computer simulation modelling; this then allows the results of the system to be tested on a model before making these changes in the real system. Once more these results shall produce further questions that may have to be considered by feeding back into the system through the feedback loops.

This model can then be used to simulate other aspects that are not studied in the previous theoretical techniques, thus improving and justifying the results of the change process to make more realistic and achievable. Again it will be necessary to investigate the project to ensure that it is making the initial requirements that were set and that all people involved are satisfied with the progress the project is taking. From this stage, Stage Five, it is felt that the Overall Technical Future State should be defined.

Stage Six is the last part of the Design Phase and involves using mainly the unprocessed culture data recorded in Stage Three. This information is directly affected on the outcomes created from Stages Four and Five, therefore all of the data collected, processed and analysed up to this point in the methodology shall be used. This Sixth stage in the methodology analyses the Current State Culture, by taking the current cultural classifications and evaluating how change and business improvements affects the business and the success of the project. This covers a number of different aspects through investigating the thoughts and opinions of those involved and how they think these changes will affect the business. The methodology explores the different ideas and the possible multiple approaches instead of pursuing a single approach. This stage understands and investigates the use of employee relations as well as empowerment and company ethics. This different approach shall again test the hypothesis to establish how to actually take the technical processed theoretical data and use that information to also consider and investigate the cultural aspect. It is this feature that is very often forgotten when transforming a system through a change process. The Sixth Stage is also very significant as it highlights the originality of the amalgamation of both the technical and cultural transformations, which are two very different aspects, but highly important factors of change.

At this point in the methodology the ideal Future State Culture shall be documented. It is also important to remember the significant differences between the 'Creating Future State' stages. These ideas are then ready to be implemented as the Optimum Future State System into the transforming current situation.

\subsection{Implementation Phase}

The Implementation Phase of the methodology is mostly about Management Techniques. The hypothesis of this research is not only to understand how to 
integrate the many business improvement techniques available to transform a system, but to test the ability to successful implement this integrated approach. This stage shall create a strategic plan of implementation from the Design Phase, by integrating a number of strategic management policies by similar scientific weighted values as that in Stage Two.

Stage Seven being the first module of the Implementation Phase starts by understanding the project definition defined within Stage One. An important factor of the methodology is that those affected by the changes are involved at every stage of the plan. Internal stakeholders have an input into the creation of the Strategic Plan by discussing the success of the Design Phase, whether the Design Phase has meet the needs of the company and whether further adjustments can be made. The next step is to assess the financial situation of the change and the relationships of the customers and the suppliers are also investigated to ensure successful implementation of the methodology. Goals and targets are set to guarantee that all aspects of the design are met, creating loops as inspection process to verify that the implementation is in line with the design. Most importantly time constraints are added to these objectives to ensure the sustainability.

Once the Strategic Plan has been documented it is analysed on topics such as costs, profits, durability, work breakdown structures and organisational work breakdown structures. The change agents are involved in the plan, the transparency and reliability of the project is also considered to ensure that the improvements are generic for other improving areas, creating a strategic plan.

Stage Eight of the methodology is to "Implement the Strategy". This module concentrates more on the monitoring of the cultural environment, by unfreezing the current culture this highlights the need for change and how this will improve the working environment

Stage Nine is seen as a revision section, it is felt that this stage will analyse the results from the implementation, all previous documented stages in the methodology are referred to and compared to the final documentation gathered at this stage. Defects can be assessed and improved upon for future projects.

The final stage, Stage Ten is considered to be one of the most important as this determines the success of the project. This stage is about controlling the project to sustain the improvements made. The first area to focus upon is understanding how to control the physical system, ensuring that the designed physical system remains within it limits and constraints. However, the system is also required to be reactive to the customer demand and the method needs to be cautiously balanced. Variances in this system need to be understood and so the actual to required measurements of the improved system need to be also be monitored and adjusted accordingly.

Not only does the physical system need to be controlled but cultural system needs to also carefully be observed. This is carried out by creating objectives to control the cultural environment, variance again will have to be measured and reduced. Measuring variance is only useful if the information is fed back into the system, by feeding this back into the system, the improvements controlled and measured will be sustained. The entire project needs to be periodically restarted as a form of continuous improvement and to emphasize sustainability and control. 


\section{Case Studies}

The methodology has been initiated within industrial environments', the flexibility of the methodology to differing but linked businesses has been tested and the outcomes are being finalised. The methodology has been applied within a supplying manufacturing environment and has been used to improve a process level production line. This case study has been used to test the methodology; the results of this preliminary implementation are limited due to the progression through the Design Methodology. This has created the project definition and an initial understanding of the current state of the production system. The data required has also been clarified, thus allowing a clear perception of what improvement techniques are applicable to the specific system. Data has been collected and the results show that there are issues related to inventory, delay periods and product transportation. It was decided to collect the data over a long period of time to ensure better accuracy of the system, through variation on product range, variety, volume and annual cyclic periods. It is often found that with Business Improvement techniques the data collection timeframe is a substantial proportion of the whole overall methodology implementation. This case study is at a point where all design and planning of the improved system is complete. These products have then been followed through into the customer and have been tested at the initiating part of the customer's production process. These case studies have enabled the hypothesis within the methodology to be successfully tested individually, within two differing manufacturing environments. This process of improvement (through the supplier and the customer) has also allowed the methodology to be tested throughout the product supply chain. With these results it has in turn allowed the methodology to be reanalysed within the supplier environment, thus understanding the effects of the supplier-buyer relationships and justifying how the hypothesis has again been tested throughout a supply chain.

\section{Conclusions}

This Design Methodology has incorporated and fulfilled a niche in the findings that were emphasised in the literature survey [3],[4]. It has integrated a number of Manufacturing Business Improvements within the Design Phase and highlighted how to apply these to a development project through weighted values of importance. The methodology has integrated both factors of change technical and cultural aspects within both phases [7]. It has also guided how to implement these findings through integrated management polices. This unified approach has ensured that a number of strategies that are not currently synchronised and assessed for project suitability can be. The literature review also highlighted that the failure rate of improvement initiative programs is quite high [6] due to lack of planning of the cultural aspects and because technical issues are easier to implement [1]. This methodology will automatically reduce factors associated to cost and time; however it also portrays as a confident view for success. Having this path of transformation alleviates cultural issues and resistance to change, it gives the project team assurance that the right 
changes are being made in the most efficient manner, therefore allowing a smoother acceptance to a change initiative program [13]. This time can be better spent on training and culture programs to ensure greater implementation success. All of these factors will aid to reducing the lead-time of a traditional change improvement program making the manufacturing environment a more competitive industry.

\section{References}

1. BALOGUN, J., \& JENKINS, M., 2003. Re-conceiving Change Management: A Knowledge-based Perspective. European Management Journal, 21, p247-257.

2. BERKHAUER-SMITH, S., BHATTI, R., \& SPEDDING, T.A., 2007. The Associations of Manufacturing Business Improvement Strategies. International Journal of Operations and Production Management. (Submitted)

3. BERKHAUER-SMITH, S., \& BHATTI, R., September 2007. Integrating both Technical And Cultural aspects of Process Improvement. $5^{\text {th }}$ International Conference on Manufacturing Research (ICMR 2007).

4. BERKHAUER-SMITH, S., \& SPEDDING, T.A., June 2007. Creating Integrated Improvement in the Manufacturing Industry. $5^{\text {th }}$ Anzam Operations Management Symposium.

5. CHIOU, E. F., HONG, J.C., HUANG, T. L., SUN, F. Y., WANG, L.J., \& YANG, S.D., 1995. Impact of employee benefits on work motivation and productivity. The International Journal of Career Management, 7, p10-14

6. CHU, K.F., 2003. An organizational culture and the empowerment for change in SMEs in the Hong Kong manufacturing industry. Journal of Materials Processing Technology, 139, p505-509.

7. LIMAN MANSAR, S., \& REIJERS, H.A., 2004. Best practices in business process redesign: an overview and qualitative evaluation of successful redesign heuristics. The International Journal of Management Science, 33, p283-306.

8. LINK, P., \& MARXK, C., 2003. Integration of risk- and chance management in the co-operation process. The International Journal of Production Economics, 90, p71-78. 